\title{
Alterations of the Gut Microbiota in Patients With Severe Chronic Heart Failure
}

\section{OPEN ACCESS}

Edited by:

Qi Zhao,

University of Science and Technology

Liaoning, China

Reviewed by:

Juan Wang,

Inner Mongolia University, China

Yanshuo Chu,

University of Texas MD Anderson

Cancer Center, United States

*Correspondence:

Ying Han

flhy81@163.com

Peng Li

58301561@qq.com

Hong Ju

hongju.hit@hotmail.com

tThese authors have contributed equally to this work

Specialty section:

This article was submitted to

Systems Microbiology,

a section of the journal

Frontiers in Microbiology

Received: 11 November 2021

Accepted: 03 December 2021

Published: 31 January 2022

Citation:

Sun W, Du D, Fu T, Han Y, Li P and Ju H (2022) Alterations of the Gut Microbiota in Patients With Severe

Chronic Heart Failure.

Front. Microbiol. 12:813289. doi: 10.3389/fmicb.2021.813289
Weiju Sun ${ }^{1+}$, Debing Du2t, Tongze Fu ${ }^{3}$, Ying Han ${ }^{4 *}$, Peng Li ${ }^{5 *}$ and Hong Ju6*

${ }^{1}$ Department of Cardiology, The First Affiliated Hospital of Harbin Medical University, Harbin, China, ${ }^{2}$ Beidahuang Industry Group General Hospital, Harbin, China, ${ }^{3}$ Harbin Medical University, Harbin, China, ${ }^{4}$ Department of Cardiology, The Fourth Affiliated Hospital of Harbin Medical University, Harbin, China, ${ }^{5}$ National Center for Biomedical Analysis, Beijing, China,

${ }^{6}$ Heilongjiang Vocational College of Biology Science and Technology, Harbin, China

Chronic heart failure (CHF) is the final outcome of almost all forms of cardiovascular diseases, remaining the main cause of mortality worldwide. Accumulating evidence is focused on the roles of gut microbial community in cardiovascular disease, but few studies have unveiled the alterations and further directions of gut microbiota in severe CHF patients. Aimed to investigate this deficiency, fecal samples from $29 \mathrm{CHF}$ patients diagnosed with NYHA Class III-IV and 30 healthy controls were collected and then analyzed using bacterial 16S rRNA gene sequencing. As a result, there were many significant differences between the two groups. Firstly, the phylum Firmicutes was found to be remarkably decreased in severe CHF patients, and the phylum Proteobacteria was the second most abundant phyla in severe CHF patients instead of phylum Bacteroides strangely. Secondly, the $\alpha$ diversity indices such as chao1, PD-whole-tree and Shannon indices were significantly decreased in the severe CHF versus the control group, as well as the notable difference in $\beta$-diversity between the two groups. Thirdly, our result revealed a remarkable decrease in the abundance of the short-chain fatty acids (SCFA)producing bacteria including genera Ruminococcaceae UCG-004, Ruminococcaceae UCG-002, Lachnospiraceae FCS020 group, Dialister and the increased abundance of the genera in Enterococcus and Enterococcaceae with an increased production of lactic acid. Finally, the alternation of the gut microbiota was presumably associated with the function including Cell cycle control, cell division, chromosome partitioning, Amino acid transport and metabolism and Carbohydrate transport and metabolism through SCFA pathway. Our findings provide the direction and theoretical knowledge for the regulation of gut flora in the treatment of severe CHF.

Keywords: severe chronic heart failure, gut microbiota, 16S rRNA gene, SCFA, patients

\section{INTRODUCTION}

Chronic heart failure (CHF) is a major health problem worldwide. It is the final outcome of almost all forms of cardiovascular diseases. CHF is recognized not only as a deregulation of hemodynamic disorder and neurocrine activation, but also an uncontrolled elevation of inflammatory responses and oxidative stress (Anker and Von Haehling, 2004; Gajarsa and Kloner, 2011; 
Hage et al., 2017; Huang et al., 2020). CHF is still associated with a high rate of hospitalization and a devastating prognosis, despite the recent development of modern combinational therapeutic strategies. Therefore, it is possible that important pathogenic mechanisms have not been targeted by current treatments, such as gut microbiota dysbiosis which have also been implicated to play a role in the development of cardiovascular diseases, including CHF (Brial et al., 2018; Jia et al., 2019; Cheng et al., 2020; Huang et al., 2020; Sanchez-Rodriguez et al., 2020).

The gut microbiota, comprising the trillions of bacteria in the gastrointestinal tract, is essential for maintaining human health in many aspects, digesting the indigestible nutrients of the host, producing vitamins and hormones, shaping the development of the mucosal immune system, and preventing the colonization of pathogenic bacteria (Amoroso et al., 2020; Sanchez-Rodriguez et al., 2020; Deledda et al., 2021). Hostmicrobiota interactions involving inflammatory and metabolic pathways have been proposed to contribute to the pathogenesis of CHF (Dantzer et al., 2018; Moshkelgosha et al., 2018; Cheng et al., 2019, 2021; Zhang et al., 2019; Kwon et al., 2020). In recent years, several sequencing-based studies have reported that the composition and function of intestinal flora between HF patients and healthy subjects are different. There are some common findings, but also considerable differences between studies (Kamo et al., 2017; Luedde et al., 2017; Cui et al., 2018; Kummen et al., 2018; Mayerhofer et al., 2018, 2020; Iqubal et al., 2020; Khan et al., 2020; Qi et al., 2021). Some computational methods have been applied in the field and other biological data (Long et al., 2021; Lv et al., 2021; Yang et al., 2021). Thus, more studies are still needed to provide detailed information on variations of gut microbial composition and its impacts on $\mathrm{CHF}$, especially the severe $\mathrm{CHF}$.

In order to define a more robust HF-related gut microbiota signature, we conducted this cross-sectional cohorts investigation. In this study, we collected stool samples from severe CHF patients and healthy controls, amplified the variable region of intestinal bacteria $16 \mathrm{~S}$ rRNA, constructed a DNA library, and then assessed the taxonomic composition of the gut microbiota in patients with severe CHF.

\section{MATERIALS AND METHODS}

\section{Study Population and Sample Collection}

Chronic heart failure patients $(n=29)$ were recruited from the First Affiliated Hospital of Harbin Medical University and the Fourth Affiliated Hospital of Harbin Medical University between April 2020 and August 2020, as well as 30 asymptomatic persons undergoing physical examinations as healthy controls. Patients who were recruited represent multiple stages of HF progression, as defined by NYHA Class III-IV. The inclusion criteria were as follows: (1) the subjects had not received antacids, probiotics, antibiotics, or antimicrobial agents within 30 days before sample collection; (2) there was no organic disease of the digestive system; and (3) they had no gastrointestinal surgery. NYHA classification was performed by patients' treating cardiologist and adjudicated by $2 \mathrm{HF}$ specialists who were blinded to the results. All patients with HF were treated according to current
HF management guidelines. Associated clinical information was collected from electronic medical records. All participants (or their direct relatives) gave written informed consent, and the First Affiliated Hospital of Harbin Medical University and the Fourth Affiliated Hospital of Harbin Medical University approved all study protocols.

We collected fresh fecal samples (each 2-5 g) from all the participants 1-2 days after admission, then transferred into sterile collecting pipes and frozen at $-80^{\circ} \mathrm{C}$ immediately.

\section{DNA Extraction and 16S rRNA Gene V3-V4 Region Sequencing}

The bacterial DNA was extracted from the fecal samples using the Tiangen stool mini kit (Tiangen, Beijing, China) according to the manufacturer's instructions. The extracted DNA from each sample was used as the template to amplify the V3-V4 region of $16 S$ rRNA genes using PCR. PCR amplification, sequencing of the PCR amplicons and quality control of raw data were performed. A sequencing library of the V3-V4 regions of the $16 \mathrm{~S}$ rRNA gene was prepared as described previously (Han et al., 2021). The purified products were mixed at an equal ratio for sequencing using an Illumina MiSeq system (Illumina Inc., United States).

\section{Statistical Analysis}

We evaluated the quality of sequencing data using the Fast-QC software $^{1}$ firstly. Next we obtained the clean data for subsequent analysis after removing the Chimera Sequence using QIIME2. ${ }^{2}$ Third, operational taxonomic units (OTUs) were delineated at the cutoff of $97 \%$ also using QIIME2, and the sequencing results were compared and analyzed to obtain the family and genus annotations of OTUs based on the Silva database. ${ }^{3}$ Then $\alpha$ - and $\beta$-diversity analyses were performed using QIIME2 fourthly. Shannon-wiener diversity index, Simpson diversity index, the observed OTUs, PD (phylogenetic diversity)-wholetree and Chaol index were evaluated. A normalized OTU abundance table was used for the $\beta$-diversity analysis, including principal coordinate analysis (PCoA) based on weighted UniFrac, and unweighted UniFrac distances. Next, we performed Lefse analysis to clarify the dominant bacteria. LEfSe is a software for discovering high-dimensional biomarkers and revealing genome characteristics. LEfSe uses linear discriminant analysis (LDA) to estimate the impact of the abundance of each component (species) on the difference effect. At last, the gene function of the sample was inferred based on the species composition obtained by sequencing, and the functional difference between different groups was analyzed using PICRUSt. ${ }^{4}$ Subsequently, the Welch's $t$-test method of two groups was performed using the STAMP software to filter the parts with $P$-value $>0.05$, and Heatmap Plot, PCA plot, and Extented error bar graphs were drawn to reveal significant differences in species abundance between different samples.

\footnotetext{
${ }^{1}$ http://www.bioinformatics.babraham.ac.uk/projects/fastqc/

${ }^{2} \mathrm{http}: / /$ qiime.org/

${ }^{3} \mathrm{https}: / /$ www.arb-silva.de/

${ }^{4}$ https://picrust.github.io/picrust/index.html
} 


\section{RESULTS}

\section{Baseline Characteristics}

The baseline characteristics of all the participants are shown in Table 1. Patients with CHF were characterized by a greater number of males, increased prevalence of comorbidity with Atrial fibrillation, worsened cardiac functions including larger left ventricular end diastolic diameter (LVEDD), decreased left ventricular ejection fraction (LVEF) and stroke volume (SV), increased E/e', coupled with increased serum Troponin I (TnI) and NT-pro B-type natriuretic peptide (NT-proBNP) levels. Patients with $\mathrm{CHF}$ also have worse renal function. Most (21) of the patients were classified to be heart failure patients with reduced ejection fraction, only 1 were classified to be heart failure patients with preserved ejection fraction, and the rest 8 of the patients were classified to be heart failure patients with midrange ejection fraction. All patients with $\mathrm{HF}$ were treated according to current HF management guidelines, including diuretics, $\beta$ blocker, Aldosterone receptor antagonist, Angiotensin converting enzyme inhibitor/Angiotensin receptor antagonist/Angiotensin receptor neprilysin inhibitor, and Sodium-glucose cotransporter 2 inhibitor.

\section{Species Classification}

The different distribution of relative abundance of top 19 at the phylum level in the two groups is presented in Figure 1A. Sequencing analysis showed that gut microbiota of the two groups were mainly classified into four phyla, including the phyla Firmicutes, Proteobacteria, Bacteroidetes, and Actinobacteria. The phylum Firmicutes was found with the highest abundance of reads in CHF patients, accounting for $59.5 \%$ in total, which was significantly decreased versus that of an abundance of $72.4 \%$ in the controls. The second was the phylum Proteobacteria, accounting for $21.3 \%$ in total, which was much more abundant versus that of an abundance of $6.9 \%$ in the controls. Likewise, bacteria belonging to the phyla Actinobacteria were more abundant in CHF patients than that in the healthy controls (2.7 vs. $0.9 \%$ ), While bacteria belonging to the Bacteroidetes phyla were slightly less abundant in HF patients than that in the healthy controls (14.9 vs. $17.7 \%$ ).

At the genus level, the microflora of CHF patients was characterized by less abundant of Faecalibacterium (10.5 vs. $22.8 \%$ ), as well as more abundant of Escherichia-Shigella (10.3 vs. $4.6 \%$ ), Enterococcus (7.7 vs. $0.0 \%$ ), and Klebsiella (6.9 vs. $1.1 \%$ ) than that in the healthy controls (Figure 1B).

\section{Analysis of $\alpha$ and $\beta$ Diversity Index}

The $\alpha$ diversity analysis was performed and then chaol curve, observed-otus curve, PD-whole-tree curve, Shannon-Wiener curve and Simpson curve based on the species annotation information were subsequently obtained by sequencing analysis. As a result, the chaol and $\mathrm{PD}$-whole-tree indices were significantly decreased in the $\mathrm{CHF}$ versus control group, as well as the Shannon indices (Figures 2A-C). The taxonomic composition of the metagenomic populations of the gut microflora samples from patients with $\mathrm{CHF}$ compared to those
TABLE 1 | Baseline characteristics of the study participants.

\begin{tabular}{|c|c|c|c|}
\hline Variables & $\begin{array}{l}\text { HF patients } \\
\qquad(n=29)\end{array}$ & $\begin{array}{l}\text { Healthy controls } \\
\qquad(n=30)\end{array}$ & $P$ value \\
\hline Age, years & $60.69(11.67)$ & $60.0(9.64)$ & 0.8062 \\
\hline Sex, male & $24(83 \%)$ & $10(33 \%)$ & $<0.0001$ \\
\hline $\mathrm{BMI}\left(\mathrm{kg} / \mathrm{m}^{2}\right)$ & $24.0(3.47)$ & $24.9(3.08)$ & 0.2849 \\
\hline NYHA class (III/IV) & 10/19 & - & - \\
\hline HFrEF/HFpEF/HFmrEF & 21/1/8 & - & - \\
\hline Hypertension & $14(48 \%)$ & $11(37 \%)$ & 0.3757 \\
\hline Diabetes & $10(34 \%)$ & $5(16.7 \%)$ & 0.1202 \\
\hline Atrial fibrillation & $10(6.7 \%)$ & $0(0)$ & 0.0003 \\
\hline Smoking & $12(41.4 \%)$ & $6(20 \%)$ & 0.0769 \\
\hline \multicolumn{4}{|c|}{ Echocardiographic parameters } \\
\hline LVEDD, mm & $62.0(8.67)$ & $43.7(3.99)$ & $<0.0001$ \\
\hline LVEF, \% & $33.8(9.1)$ & $63.2(4.65)$ & $<0.0001$ \\
\hline SV, ml & $46.7(11.1)$ & $68.9(11.94)$ & $<0.0001$ \\
\hline$E / e^{\prime}$ & $19.3(6.5)$ & $13.4(3.1)$ & $<0.0001$ \\
\hline \multicolumn{4}{|l|}{ Laboratory parameters } \\
\hline Tnl, ng/dL & $\begin{array}{c}55.87 \\
(0.10-88.7)\end{array}$ & $0.012(0-0.048)$ & 0.0007 \\
\hline NT-proBNP, pg/mL & $\begin{array}{c}4745.7 \\
(1130-16755)\end{array}$ & $124.0(25-258)$ & $<0.0001$ \\
\hline Leukocyte, $10^{9} / \mathrm{L}$ & $7.2(3.00)$ & $6.7(1.74)$ & 0.4366 \\
\hline Neutrophils, $10^{9} / \mathrm{L}$ & $4.9(2.51)$ & $4.0(1.30)$ & 0.0878 \\
\hline Lymphocytes, 109/L & $1.63(0.61)$ & $2.1(0.65)$ & 0.0110 \\
\hline Monocyte, $10^{9} / \mathrm{L}$ & $0.5(0.18)$ & $0.4(0.14)$ & 0.0632 \\
\hline Hemoglobin, g/L & $140.7(26.53)$ & $140.8(17.04)$ & 0.9834 \\
\hline BUN, mg/dl & $8.0(3.04)$ & $5.5(1.81)$ & 0.0003 \\
\hline Serum creatinine, mg/dl & $87.4(35.16)$ & $67.4(18.35)$ & 0.0078 \\
\hline Fast glucose & $6.5(3.67)$ & $5.2(1.30)$ & 0.0698 \\
\hline Cholesterol & $4.2(0.96)$ & $5.0(0.90)$ & 0.0013 \\
\hline Triglycerides & $1.5(0.92)$ & $1.9(1.66)$ & 0.2305 \\
\hline HDL-C & $0.9(0.22)$ & $0.91(0.22)$ & 0.8917 \\
\hline LDL-C & $2.5(0.87)$ & $2.9(0.80)$ & 0.0592 \\
\hline Treatment diuretics & 29 (100\%) & - & - \\
\hline$\beta$ blocker & 27 (93\%) & - & - \\
\hline MRA & 29 (100\%) & - & - \\
\hline ACEI/ARB/ARNI & $26(90 \%)$ & - & - \\
\hline SGLT2i & $21(72 \%)$ & - & - \\
\hline
\end{tabular}

Results are presented as median (with standard error or upper and lower quartiles) or\% where appropriate. BMI, body mass index; NYHA, New York Heart Association; HFrEF, heart failure with reduced EF; HFpEF, heart failure with preserved EF; HfmrEF, heart failure with midrange EF; LVEDD: left ventricular end diastolic diameter; LVEF: Left ventricular ejection fraction; Thl, Troponin I; NT-proBNP: NT-pro B-type natriuretic peptide; HDL-C, high density lipoproteincholesterol; LDL-C, low density lipoprotein-cholesterol; MRA, Aldosterone receptor antagonist; ACEl, Angiotensin converting enzyme inhibitor; ARB, Angiotensin receptor antagonist; ARNI, Angiotensin receptor neprilysin inhibitor; SGLT2i, Sodium-glucose cotransporter 2 inhibitor.

from the healthy control group were also analyzed using Principal Coordinate Analysis (PCoA). The differences in $\beta$-diversity based on the weighted UniFrac between the HF and healthy control groups were also shown in Figure 2D, which indicates that the fecal microbial structure in the CHF group was obviously different than that of the healthy control group in condition of the presence of OTU. 

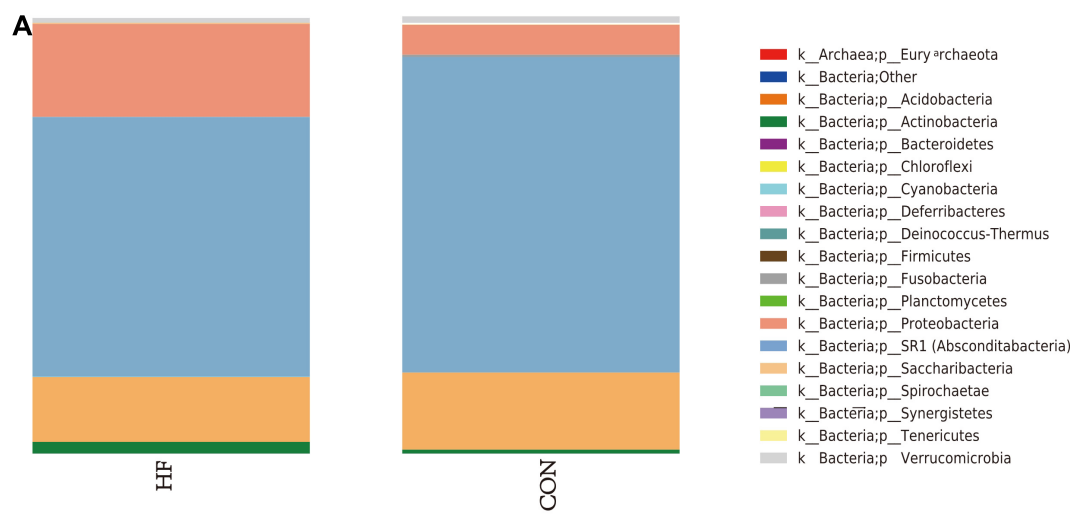

B

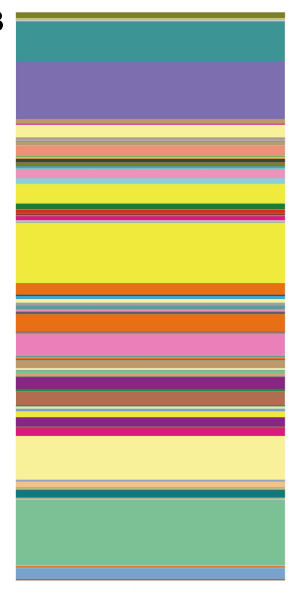

早

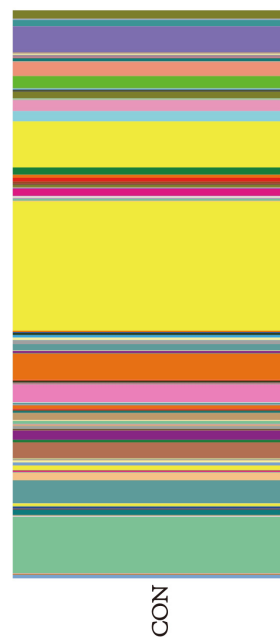

k_Bacteria;p_Firmicutes;c_Bacilli;o_Lactobacillales;f_Enterococcaceae;g_Enterococcus

_Bacteria;P_Firmicutes;c_Bacilli;0_Lactobacillales;f_Lactobacillaceae;g_Lactobacillus

k_Bacteria;p_Firmicutes;c_Bacilli;0_Lactobacillales;f_Streptococcaceae;g Lactococcus

k_Bacteria;p_Actinobacteria;c_Actinobacteria;0_Actinomycetales;f_Actinomycetaceae;0ther

- k_Bacteria;p_Actinobacteria;c_Actinobacteria;0_Actinomycetales;f_Actinomycetaceae;g_Actinomyces

k_Bacteria; $p \_$Actinobacteria;c_Actinobacteria;o_Actinomycetales;f_Actinomycetaceae;g_Mobiluncus

— k_Bacteria;p_Actinobacteria;c_Actinobacteria;0_Actinomycetales;f_Actinomycetaceae;g_uncultured

_ k_Bacteria;P_Actinobacteria;c_Actinobacteria;0_Bifidobacteriales;f_Bifidobacteriaceae;Other

- k_Bacteria;P_Actinobacteria;c_Actinobacteria;0_Bifidobacteriales;f_Bifidobacteriaceae;g_Bifidobacterium

_ K_Bacteria;p_Proteobacteria;c_Gammaproteobacteria;0_Enterobacteriales;f_Enterobacteriaceae;g_Raoultella

— k_Bacteria;p_Proteobacteria;c_Gammaproteobacteria;o_Enterobacteriales;f_Enterobacteriaceae;g_Salmonella

- k_Bacteria;p_Proteobacteria;c_Gammaproteobacteria;o_Enterobacteriales;f_Enterobacteriaceae;g_Serratia

- k_Bacteria;p_Synergistetes; $c$ Synergistia;0_Synergistales; $;$ _Synergistaceae;g_Cloacibacillus

- k_Bacteria;p_Synergistetes;c_Synergistia;0_Synergistales;f_Synergistaceae;g_Fretibacterium

_ k_Bacteria;p_Synergistetes;c_Synergistia;0_Synergistales;f_Synergistaceae;g_Jonquetella

— k_Bacteria;p_Synergistetes;c_Synergistia;0_Synergistales;f_Synergistaceae;g_Pyramidobacter

- k_Bacteria;p_Firmicutes;c_Clostridia;0_Clostridiales;f_Family XI;g_Ezakiella

k_Bacteria;p_Firmicutes;c_Clostridia;0_Clostridiales;f_Family XI;g_Finegoldia

_ k_Bacteria;p_Firmicutes;c_Clostridia;0_Clostridiales;f_Family XI;g_Gallicola

_. k_Bacteria;p_Firmicutes;c_Clostridia;0_Clostridiales;f_Family XI;g_Gottschalkia

_. k_Bacteria;p_Firmicutes;c_Clostridia;0_Clostridiales;f_Family XI;g_Helcococcus

- $\mathrm{k}$ Bacteria;p Firmicutes;c Clostridia;0 Clostridiales; Family XI;g Murdochiella

FIGURE 1 | The distribution of relative abundance in the CHF and the healthy control group. Panel (A) shows the distribution of relative abundance of top 19 at phylum level in the two groups. Panel (B) shows the distribution of relative abundance at the genus level in the two groups. HF, CHF group; CON, the healthy control group.

\section{Analysis of Differential Taxonomy Expression}

A differential taxonomy expression analysis was performed using limma algorithms, focusing on differences at the genus level (Figure 3). There was a remarkable difference with 152 generus in fecal microflora between the CHF and healthy control group in our result. Among these changes, the decreased abundance of the genera Ruminococcaceae UCG-004, Ruminococcaceae UCG002, Lachnospiraceae FCS020 group, Dialister and the increased abundance of the genera in Enterococcus and Enterococcaceae were the most notable features (Figure 3B).

\section{Analysis of Predictive Function}

Based on the closed-reference OUTs, PICRUSt was utilized to predict abundances of the functional category COG orthologs (COs) and KEGG orthologs (KOs). Some of these COs and KOs were indicated to be significantly different in fecal microbiomes between the CHF and healthy control group $(P<0.05 ;$ Figure 4). Furthermore, there were also some meaningful results related with the function including cell cycle control, cell division, chromosome partitioning, Inorganic ion transport and metabolism, translation, ribosomal structure and biogenesis, amino acid transport and metabolism and carbohydrate transport and metabolism.

\section{DISCUSSION}

In the current study, bacterial $16 \mathrm{~S}$ rRNA gene sequencing was applied to confirm the composition and differential expression in gut microbiota between 29 severe CHF patients and 30 healthy controls, resulting in a number of notable differences between these two groups. Firstly, the phylum Proteobacteria was significantly more abundant in CHF patients than controls, whereas the phylum Firmicutes was found remarkably decreased in CHF patients. Secondly, the $\alpha$ diversity indices significantly decreased in the CHF versus control group, as well as the notable differences in $\beta$-diversity between the $\mathrm{CHF}$ and healthy control groups, which indicates that the fecal microbial structure in the $\mathrm{CHF}$ group was obviously different than that of the healthy control group in 

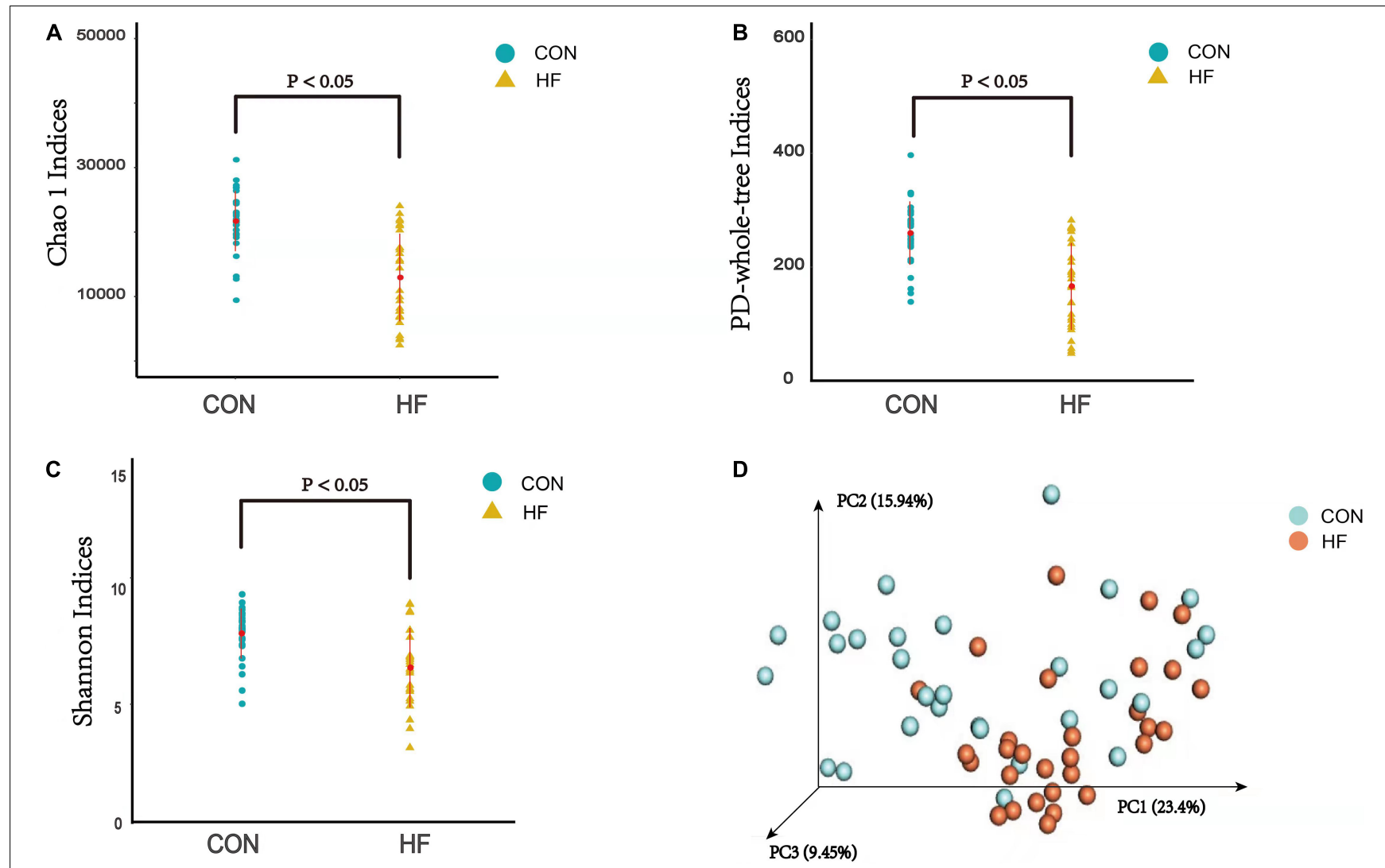

FIGURE 2 | The $\alpha$-diversity indices and the $\beta$-diversity of the gut microflora between the CHF group and the healthy control group. (A) PD-whole tree indice; (B) Chao1 indices; (C) Shannon indices; (D) $\beta$-diversity based on the weighted UniFrac. HF, CHF group; CON, the healthy control group.

condition of the presence of OTU. Thirdly, the microflora of $\mathrm{CHF}$ patients was characterized by the decreased abundance of the genera Ruminococcaceae UCG-004, Ruminococcaceae UCG-002, Lachnospiraceae FCS020 group, Dialister and the increased abundance of the genera in Enterococcus and Enterococcaceae. Finally, the alternation of the gut microbiota was presumably associated with the function including cell cycle control, cell division, chromosome partitioning, inorganic ion transport and metabolism, translation, ribosomal structure and biogenesis, amino acid transport and metabolism and carbohydrate transport and metabolism. To our knowledge, this is the first study to explore the changes in the gut flora of patients with severe $\mathrm{CHF}$.

Tang et al. (2017) put forward the "gut hypothesis of heart failure" for the first time. The hypothesis implies that reduced cardiac output caused by heart failure can lead to decreased intestinal perfusion, mucosal ischemia, and then intestinal mucosal destruction. These changes in the intestinal barrier function, in turn, can lead to increased intestinal permeability, intestinal malnutrition, bacterial translocation and increased circulating endotoxins, resulting in the potential inflammation associated with HF (Nagatomo and Tang, 2015; Tang et al., 2017, 2019; Harikrishnan, 2019). There are also several studies that have reported the composition and function of intestinal flora between HF patients and healthy subjects are different (Kamo et al., 2017; Luedde et al., 2017; Cui et al., 2018; Mayerhofer et al., 2018, 2020). Likewise, we found significant differences in the composition of fecal microbes between CHF patients and healthy controls, suggesting that there is a link between intestinal microflora disorders and CHF. At the phylum level, Firmicutes and Bacteroides are the two most abundant phyla in the healthy intestine. They are closely related to environmental conditions and may be beneficial or problematic for human and animal health. However, the phylum Firmicutes was remarkably decreased in severe CHF patients simultaneously in our study. What is more, the phylum Proteobacteria was the second most abundant phyla in severe CHF patients instead of phylum Bacteroides. All the members of the phylum Proteobacteria are Gram-negative bacteria, with the outer membrane mainly composed by lipopolysaccharide (LPS). Most of the phylum Proteobacteria are pathogenic bacteria, as a result, it is considered to be a microbial signature of dysbiosis in gut microbiota (Shin et al., 2015). Furthermore, LPS leaking into the blood through the intestinal wall can stimulate the production of a variety of pro-inflammatory cytokines, which are involved in the apoptosis, hypertrophy and fibrosis of heart cells, playing an important role in the occurrence and development of heart failure (Sandek et al., 2012).

Microbiota diversity has been considered to be a new health biomarker (Shanahan, 2010; Tang et al., 2018; Aponte et al., 2020; 


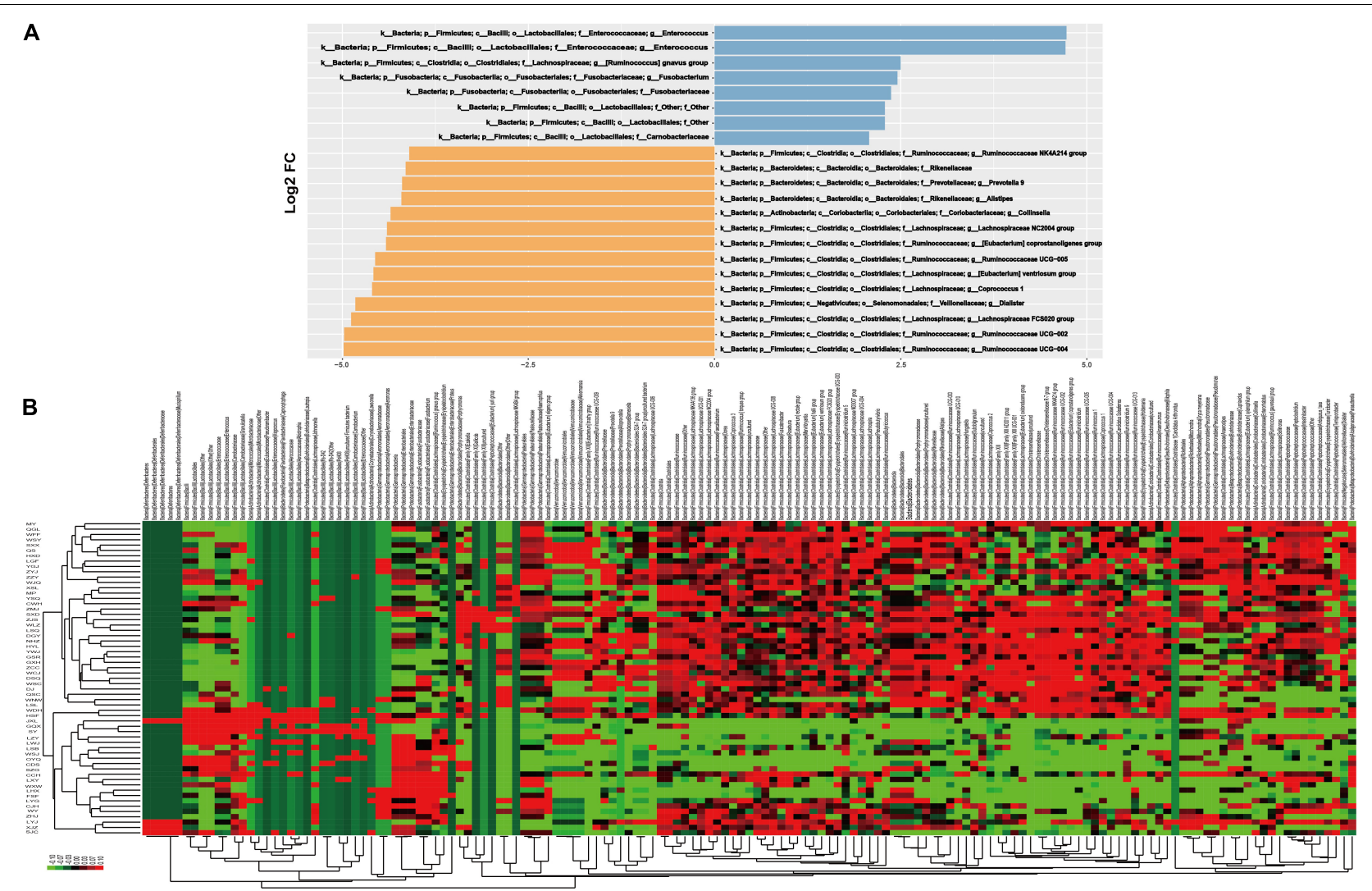

FIGURE 3 | The results of differential taxonomy expression analysis using limma algorithm. Panel (A) shows the differential taxonomy expression in the CHF patients vs. healthy control group; (B) shows the differential taxonomy expression in every samples.

Nadia and Ramana, 2020; Mulpuru et al., 2021). The $\alpha$ diversity index mainly focuses on the number of species in a local uniform habitat, reflecting the abundance and diversity of the microbial community. As the indicators of the community richness, the chaol and $\mathrm{PD}$-whole-tree indices were significantly decreased in the CHF patients versus control group, as well as the Shannon indices, which is an important index used to estimate the microbial diversity. $\beta$-diversity is a comparative analysis of the microbial community composition of different groups of samples, constituting the overall diversity together with $\alpha$ diversity. These significantly different indexes in the current study revealed that loss of gut flora biodiversity is associated with CHF.

In the further differential classification expression analysis using the limma algorithm, we are more focused on the differences in the genus level. Consistent with other experimental results (Kummen et al., 2018; Mayerhofer et al., 2020), the decreased abundance of the genera Ruminococcaceae and Lachnospiraceae were also discovered in our study, which are known for their ability to synthesize short-chain fatty acids (SCFA) through the fermentation of dietary polysaccharides. In addition, we firstly reported that the decrease of genra Dialister and the increase of the genera in Enterococcus and Enterococcaceae were also the most notable features in $\mathrm{CHF}$ patients. Dialister is one of the most representative types of intestinal flora associated with irritable bowel syndrome (Lopetuso et al., 2018). The main products are lactic acid, acetic acid and formic acid, and those are also SCFAs. Enterococcus and Enterococcaceae (Byappanahalli et al., 2012; Gouba et al., 2020; Xie et al., 2021) are conditional pathogens, causing infections such as urinary tract infections, purulent abdominal infections, sepsis, endocarditis and diarrhea. On the other hand, their microbial preparations can enhance the activity of macrophage cells and promote the immune response. At the same time, due to their metabolism to produce lactic acid, they can form a lactic acid barrier to resist foreign pathogenic microorganisms. The above results suggested that the intestinal flora may participate in the occurrence and development of heart failure through the action of SCFAs. As we all know, SCFAs play an important role in the regulation of inflammation, which is definitely involved in the pathophysiological process of CHF. SCFAs can reduce the production of inflammatory factors by activating GPR41/43 (Li et al., 2018; Weber et al., 2018; Onyszkiewicz et al., 2019). Acetic acid may reduce the production of interleukin-6 and interleukin-8 (Sah et al., 2013; Yu et al., 2015), and butyric acid and propionic acid can reduce the production of interleukin-6 (Esquivel-Rendon et al., 2019; Yue et al., 2020). Butyrate plays an anti-inflammatory role through inducing Foxp3 + Treg cell proliferation and suppressing the generation of Th17 cells by activating $\mathrm{G}$ protein-coupled receptor 


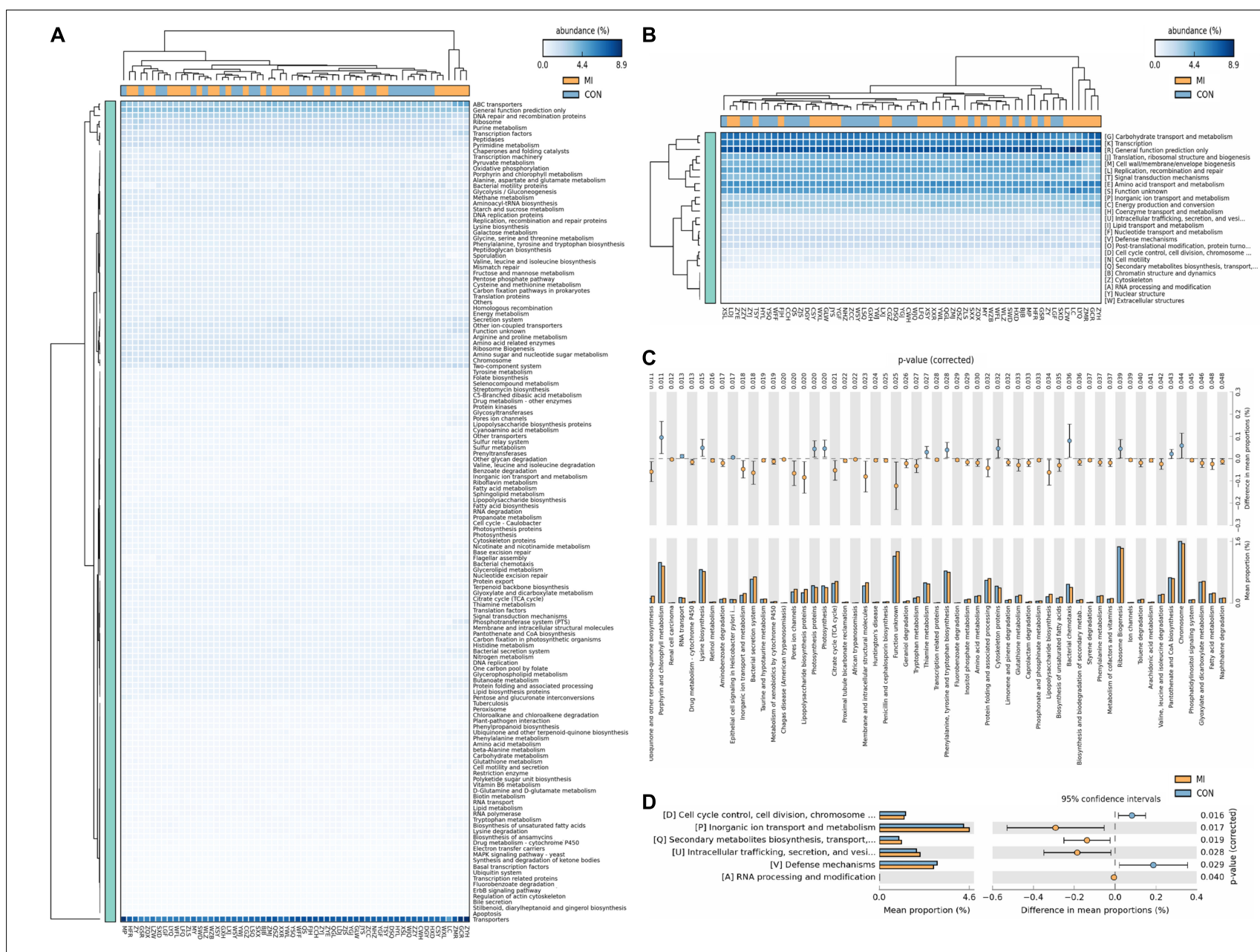

FIGURE 4 | The results of PICRUst based on closed-reference OTU to predict the abundances of functional categories COG orthologs (COs) and KEGG orthologs (KOs). Panels (A,C) shows the KOs with significantly different abundances in the fecal microbiome between the CHF group and healthy control group; (B,D) shows the COs with significantly different abundances in the fecal microbiome between the CHF group and healthy control group. HF, CHF group; CON, the healthy control group.

43 (Sivaprakasam et al., 2016; Bhaskaran et al., 2018). Other studies have also shown that SCFAs can also improve insulin sensitivity, regulate fat and muscle energy metabolism, and play an important role in the development of diabetes and obesity (Canfora et al., 2015; Muller et al., 2019).

The consequent results from analysis of predictive function using PICRUSt revealed several functional pathways involved in the relationship between gut microbiomes and CHF, including cell cycle control, cell division, chromosome partitioning, inorganic ion transport and metabolism, ribosomal structure and biogenesis, amino acid transport and metabolism and carbohydrate transport and metabolism. The occurrence and development of heart failure are inseparable from the disorders of carbohydrate metabolism, amino acids metabolism and lipid metabolism. The aforementioned differential classification expression analysis has indicated a notable reduction in SCFAproducing bacteria in patients with severe CHF. As a matter of fact, SCFAs can not only modulate the carbohydrate metabolism through activating $G$ protein-coupled receptor and
AMP-activated protein kinase, improving insulin sensitivity (Gao et al., 2009; den Besten et al., 2013), but also increasing the production of ketogenic amino acids and ketone bodies (Thevenet et al., 2016; Pujol et al., 2018), which is considered to be one of the energy sources of failing myocardium and closely related to the process of heart failure. Furthermore, as a histone deacetylase inhibitor, SCFAs can partly regulate cell proliferation, apoptosis and differentiation by inhibiting histone deacetylase, as well as exert anti-inflammatory effects (Koh et al., 2016; Makki et al., 2018; Alrafas et al., 2020).

Some limitations should be acknowledged. First, although all participants were from the same region, experienced a normal/routine lifestyle and had similar nutritional patterns, including typical Chinese diets based on carbohydrates versus high-fat diets, and participated in routine levels of general physical activity (e.g., housework and walking), we were still unable to completely account for the influence of diet on gut microbiota. Second, the enrolled cohort was a small sample size and predominantly male in patients with severe CHF. 
Further studies informing the generalizability of gut microbiota in patients with CHF are warranted. Third, it is also necessary to address temporality of associations between gut dysbiosis and CHF. Finally, multiple omics data, such as metabolomics and proteomics, will be warranted to confirm the suppose that SCFAs produced by the gut microbiota participating the pathophysiological processes of $\mathrm{CHF}$ and explore the exact mechanisms.

In conclusion, the current results firstly exhibited remarkable differencesion the composition and diversity of the gut flora of severe CHF patients and healthy controls using bacterial $16 \mathrm{~S}$ rRNA gene sequencing. The microflora of severe $\mathrm{CHF}$ patients was characterized by the decreased abundance of the SCFA-producing bacteria including genera Ruminococcaceae UCG-004, Ruminococcaceae UCG-002, Lachnospiraceae FCS020 group, Dialister and the increased abundance of the genera in Enterococcus and Enterococcaceae with an increased production of lactic acid. Moreover, the alternation of the gut microbiota was presumably associated with the function including cell cycle control, cell division, chromosome partitioning, amino acid transport and metabolism and carbohydrate transport and metabolism through SCFA pathway. This information may not only improve our understanding of the pathogenesis of severe $\mathrm{CHF}$, but also suggest that the regulation of the composition of gut microbiota may represent a promising therapeutic target.

\section{DATA AVAILABILITY STATEMENT}

The datasets presented in this study can be found in online repositories. The names of the repository/repositories

\section{REFERENCES}

Alrafas, H. R., Busbee, P. B., Chitrala, K. N., Nagarkatti, M., and Nagarkatti, P. (2020). Alterations in the gut microbiome and suppression of histone deacetylases by resveratrol are associated with attenuation of colonic inflammation and protection against colorectal Cancer. J. Clin. Med. 9:1796. doi: $10.3390 /$ jcm 9061796

Amoroso, C., Perillo, F., Strati, F., Fantini, M. C., Caprioli, F., and Facciotti, F. (2020). the role of gut microbiota biomodulators on mucosal immunity and intestinal inflammation. Cells 9:1234. doi: 10.3390/cells9051234

Anker, S. D., and Von Haehling, S. (2004). Inflammatory mediators in chronic heart failure: an overview. Heart 90, 464-470. doi: 10.1136/hrt.2002.007005

Aponte, M., Murru, N., and Shoukat, M. (2020). Therapeutic, prophylactic, and functional use of probiotics: a current perspective. Front. Microbiol. 11:562048. doi: 10.3389/fmicb.2020.562048

Bhaskaran, N., Quigley, C., Paw, C., Butala, S., Schneider, E., and Pandiyan, P. (2018). Role of short chain fatty acids in controlling tregs and immunopathology during mucosal infection. Front. Microbiol. 9:1995. doi: 10. 3389/fmicb.2018.01995

Brial, F., Le Lay, A., Dumas, M. E., and Gauguier, D. (2018). Implication of gut microbiota metabolites in cardiovascular and metabolic diseases. Cell Mol. Life Sci. 75, 3977-3990. doi: 10.1007/s00018-018-2901-1

Byappanahalli, M. N., Nevers, M. B., Korajkic, A., Staley, Z. R., and Harwood, V. J. (2012). Enterococci in the environment. Microbiol. Mol. Biol. Rev. 76, 685-706.

Canfora, E. E., Jocken, J. W., and Blaak, E. E. (2015). Short-chain fatty acids in control of body weight and insulin sensitivity. Nat. Rev. Endocrinol. 11, 577-591. doi: 10.1038/nrendo.2015.128

Cheng, L., Qi, C., Yang, H., Lu, M., Cai, Y., Fu, T., et al. (2021). gutMGene: a comprehensive database for target genes of gut microbes and microbial metabolites. Nucleic Acids Res. gkab786. doi: 10.1093/nar/gkab786 and accession number(s) can be found in the article/ supplementary material.

\section{ETHICS STATEMENT}

The studies involving human participants were reviewed and approved by The First Affiliated Hospital of Harbin Medical University and The Fourth Affiliated Hospital of Harbin Medical University approved all study protocols. The patients/participants provided their written informed consent to participate in this study.

\section{AUTHOR CONTRIBUTIONS}

$\mathrm{YH}, \mathrm{PL}$, and $\mathrm{HJ}$ conceived and designed the experiments. $\mathrm{YH}$ and TF analyzed data and drew the pictures. WS and DD wrote this manuscript. All authors read and approved the final manuscript.

\section{FUNDING}

The National Key Research and Development Program of China (2019YFC0118100), National Natural Science Foundation of China (82100353, 81671760, and 81873910), and Excellent youth project of the Fourth Affiliated Hospital of Harbin Medical University (No. HYDSYYXQN202007).

Cheng, L., Qi, C., Zhuang, H., Fu, T., and Zhang, X. (2020). gutMDisorder: a comprehensive database for dysbiosis of the gut microbiota in disorders and interventions. Nucleic Acids Res. 48, D554-D560.

Cheng, L., Yang, H., Zhao, H., Pei, X., Shi, H., Sun, J., et al. (2019). MetSigDis: a manually curated resource for the metabolic signatures of diseases. Brief. Bioinform. 20, 203-209. doi: 10.1093/bib/bbx103

Cui, X., Ye, L., Li, J., Jin, L., Wang, W., Li, S., et al. (2018). Metagenomic and metabolomic analyses unveil dysbiosis of gut microbiota in chronic heart failure patients. Sci. Rep. 8:635. doi: 10.1038/s41598-017-18756-2

Dantzer, R., Cohen, S., Russo, S. J., and Dinan, T. G. (2018). Resilience and immunity. Brain Behav. Immun. 74, 28-42. doi: 10.1016/j.bbi.2018.08.010

Deledda, A., Annunziata, G., Tenore, G. C., Palmas, V., Manzin, A., and Velluzzi, F. (2021). Diet-derived antioxidants and their role in inflammation, obesity and gut microbiota modulation. Antioxidants 10:708. doi: 10.3390/antiox10050708

den Besten, G., Lange, K., Havinga, R., Van Dijk, T. H., Gerding, A., Van Eunen, K., et al. (2013). Gut-derived short-chain fatty acids are vividly assimilated into host carbohydrates and lipids. Am. J. Physiol. Gastrointest. Liver Physiol. 305, G900-G910. doi: 10.1152/ajpgi.00265.2013

Esquivel-Rendon, E., Vargas-Mireles, J., Cuevas-Olguin, R., Miranda-Morales, M., Acosta-Mares, P., Garcia-Oscos, F., et al. (2019). Interleukin 6 dependent synaptic plasticity in a social defeat-susceptible prefrontal cortex circuit. Neuroscience 414, 280-296. doi: 10.1016/j.neuroscience.2019.07.002

Gajarsa, J. J., and Kloner, R. A. (2011). Left ventricular remodeling in the postinfarction heart: a review of cellular, molecular mechanisms, and therapeutic modalities. Heart. Fail Rev. 16, 13-21. doi: 10.1007/s10741-010-9181-7

Gao, Z., Yin, J., Zhang, J., Ward, R. E., Martin, R. J., Lefevre, M., et al. (2009). Butyrate improves insulin sensitivity and increases energy expenditure in mice. Diabetes 58, 1509-1517. doi: 10.2337/db08-1637

Gouba, N., Yimagou, E. K., Hassani, Y., Drancourt, M., Fellag, M., and Mbogning Fonkou, M. D. (2020). Enterococcus burkinafasonensis sp. nov. isolated from 
human gut microbiota. New Microbes New Infect. 36:100702. doi: 10.1016/j. nmni.2020.100702

Hage, C., Michaelsson, E., Linde, C., Donal, E., Daubert, J. C., Gan, L. M., et al. (2017). Inflammatory biomarkers predict heart failure severity and prognosis in patients with heart failure with preserved ejection fraction: a holistic proteomic approach. Circ. Cardiovasc. Genet. 10:e001633. doi: 10.1161/CIRCGENETICS. 116.001633

Han, Y., Gong, Z., Sun, G., Xu, J., Qi, C., Sun, W., et al. (2021). Dysbiosis of gut microbiota in patients with acute myocardial infarction. Front. Microbiol. 12:680101. doi: 10.3389/fmicb.2021.680101

Harikrishnan, S. (2019). Diet, the gut microbiome and heart failure. Card Fail Rev. 5, 119-122. doi: 10.15420/cfr.2018.39.2

Huang, S. Y., Xiang, X., Qiu, L., Wang, L. Y., Zhu, B. H., Guo, R. Q., et al. (2020). Transfection of TGF-beta shRNA by using ultrasound-targeted microbubble destruction to inhibit the early adhesion repair of rats wounded achilles tendon in vitro and in vivo. Curr. Gene Ther. 20, 71-81. doi: 10.2174/ 1566523220666200516165828

Iqubal, A., Iqubal, M. K., Khan, A., Ali, J., Baboota, S., and Haque, S. E. (2020). Gene therapy, a novel therapeutic tool for neurological disorders: current progress, challenges and future prospective. Curr. Gene Ther. 20, 184-194. doi: 10.2174/ 1566523220999200716111502

Jia, Q., Xie, Y., Lu, C., Zhang, A., Lu, Y., Lv, S., et al. (2019). Endocrine organs of cardiovascular diseases: gut microbiota. J. Cell Mol. Med. 23, 2314-2323. doi: $10.1111 /$ jcmm.14164

Kamo, T., Akazawa, H., Suda, W., Saga-Kamo, A., Shimizu, Y., Yagi, H., et al. (2017). Dysbiosis and compositional alterations with aging in the gut microbiota of patients with heart failure. PLoS One 12:e0174099. doi: 10.1371/ journal.pone.0174099

Khan, A., Zahra, A., Mumtaz, S., Fatmi, M. Q., and Khan, M. J. (2020). Integrated in-silico analysis to study the role of microRNAs in the detection of chronic kidney diseases. Curr. Bioinform. 15, 144-154. doi: 10.2174/ 1574893614666190923115032

Koh, A., De Vadder, F., Kovatcheva-Datchary, P., and Backhed, F. (2016). From dietary fiber to host physiology: short-chain fatty acids as key bacterial metabolites. Cell 165, 1332-1345. doi: 10.1016/j.cell.2016.05.041

Kummen, M., Mayerhofer, C. C. K., Vestad, B., Broch, K., Awoyemi, A., StormLarsen, C., et al. (2018). Gut microbiota signature in heart failure defined from profiling of 2 independent cohorts. J. Am. Coll. Cardiol. 71, 1184-1186. doi: 10.1016/j.jacc.2017.12.057

Kwon, E., Cho, M., Kim, H., and Son, H. S. (2020). A study on host tropism determinants of influenza virus using machine learning. Curr. Bioinform. 15, 121-134. doi: 10.2174/1574893614666191104160927

Li, M., Van Esch, B., Henricks, P. A. J., Folkerts, G., and Garssen, J. (2018). The antiinflammatory effects of short chain fatty acids on lipopolysaccharide- or tumor necrosis factor alpha-stimulated endothelial cells via activation of GPR41/43 and inhibition of HDACs. Front. Pharmacol. 9:533. doi: 10.3389/fphar.2018. 00533

Long, J., Yang, H., Yang, Z., Jia, Q., Liu, L., Kong, L., et al. (2021). Integrated biomarker profiling of the metabolome associated with impaired fasting glucose and type 2 diabetes mellitus in large-scale Chinese patients. Clin. Transl. Med. 11:e432. doi: $10.1002 / \mathrm{ctm} 2.432$

Lopetuso, L. R., Petito, V., Graziani, C., Schiavoni, E., Paroni Sterbini, F., Poscia, A., et al. (2018). Gut microbiota in health, diverticular disease, irritable bowel syndrome, and inflammatory bowel diseases: time for microbial marker of gastrointestinal disorders. Dig. Dis. 36, 56-65. doi: 10.1159/00047 7205

Luedde, M., Winkler, T., Heinsen, F. A., Ruhlemann, M. C., Spehlmann, M. E., Bajrovic, A., et al. (2017). Heart failure is associated with depletion of core intestinal microbiota. ESC Heart Fail 4, 282-290. doi: 10.1002/ehf2.12155

Lv, H., Dao, F. Y., Zulfiqar, H., and Lin, H. (2021). DeepIPs: comprehensive assessment and computational identification of phosphorylation sites of SARSCoV-2 infection using a deep learning-based approach. Brief. Bioinform. 22:bbab244. doi: 10.1093/bib/bbab244

Makki, K., Deehan, E. C., Walter, J., and Backhed, F. (2018). The impact of dietary fiber on gut microbiota in host health and disease. Cell Host Microbe 23, 705-715. doi: 10.1016/j.chom.2018.05.012

Mayerhofer, C. C. K., Awoyemi, A. O., Moscavitch, S. D., Lappegard, K. T., Hov, J. R., Aukrust, P., et al. (2018). Design of the GutHeart-targeting gut microbiota to treat heart failure-trial: a Phase II, randomized clinical trial. ESC Heart Fail 5, 977-984. doi: 10.1002/ehf2.12332

Mayerhofer, C. C. K., Kummen, M., Holm, K., Broch, K., Awoyemi, A., Vestad, B., et al. (2020). Low fibre intake is associated with gut microbiota alterations in chronic heart failure. ESC Heart Fail 7, 456-466. doi: 10.1002/ehf2.12596

Moshkelgosha, S., Masetti, G., Berchner-Pfannschmidt, U., Verhasselt, H. L., Horstmann, M., Diaz-Cano, S., et al. (2018). Gut microbiome in BALB/c and C57BL/6J mice undergoing experimental thyroid autoimmunity associate with differences in immunological responses and thyroid function. Horm. Metab. Res. 50, 932-941. doi: 10.1055/a-0653-3766

Muller, M., Hernandez, M. A. G., Goossens, G. H., Reijnders, D., Holst, J. J., Jocken, J. W. E., et al. (2019). Circulating but not faecal short-chain fatty acids are related to insulin sensitivity, lipolysis and GLP-1 concentrations in humans. Sci. Rep. 9:12515. doi: 10.1038/s41598-019-48775-0

Mulpuru, V., Semwal, R., Varadwaj, P. K., and Mishra, N. (2021). HAMP: a knowledgebase of antimicrobial peptides from human microbiome. Curr. Bioinform. 16, 534-540. doi: 10.2174/1574893615999200802041228

Nadia, and Ramana, J. (2020). The human OncoBiome database: a database of cancer microbiome datasets. Curr. Bioinform. 15, 472-477. doi: 10.2174/ 1574893614666190902152727

Nagatomo, Y., and Tang, W. H. (2015). Intersections between microbiome and heart failure: revisiting the gut hypothesis. J. Card Fail 21, 973-980. doi: 10. 1016/j.cardfail.2015.09.017

Onyszkiewicz, M., Gawrys-Kopczynska, M., Konopelski, P., Aleksandrowicz, M., Sawicka, A., Kozniewska, E., et al. (2019). Butyric acid, a gut bacteria metabolite, lowers arterial blood pressure via colon-vagus nerve signaling and GPR41/43 receptors. Pflugers Arch. 471, 1441-1453. doi: 10.1007/s00424-019-02322-y

Pujol, J. B., Christinat, N., Ratinaud, Y., Savoia, C., Mitchell, S. E., and Dioum, E. H. M. (2018). Coordination of GPR40 and ketogenesis signaling by medium chain fatty acids regulates beta cell function. Nutrients 10:473. doi: 10.3390/ nu10040473

Qi, C., Wang, P., Fu, T., Lu, M., Cai, Y., Chen, X., et al. (2021). A comprehensive review for gut microbes: technologies, interventions, metabolites and diseases. Brief. Funct. Genomics 20, 42-60. doi: 10.1093/bfgp/elaa029

Sah, S. K., Kim, B. H., Park, G. T., Kim, S., Jang, K. H., Jeon, J. E., et al. (2013). Novel isonahocol E(3) exhibits anti-inflammatory and anti-angiogenic effects in endothelin-1-stimulated human keratinocytes. Eur. J. Pharmacol. 720, 205-211. doi: 10.1016/j.ejphar.2013.09.077

Sanchez-Rodriguez, E., Egea-Zorrilla, A., Plaza-Diaz, J., Aragon-Vela, J., MunozQuezada, S., Tercedor-Sanchez, L., et al. (2020). The gut microbiota and its implication in the development of atherosclerosis and related cardiovascular diseases. Nutrients 12:605. doi: 10.3390/nu12030605

Sandek, A., Bjarnason, I., Volk, H. D., Crane, R., Meddings, J. B., Niebauer, J., et al. (2012). Studies on bacterial endotoxin and intestinal absorption function in patients with chronic heart failure. Int. J. Cardiol. 157, 80-85. doi: 10.1016/j. ijcard.2010.12.016

Shanahan, F. (2010). Probiotics in perspective. Gastroenterology 139, 1808-1812. doi: $10.1053 /$ j.gastro.2010.10.025

Shin, N. R., Whon, T. W., and Bae, J. W. (2015). Proteobacteria: microbial signature of dysbiosis in gut microbiota. Trends Biotechnol. 33, 496-503. doi: 10.1016/j. tibtech.2015.06.011

Sivaprakasam, S., Prasad, P. D., and Singh, N. (2016). Benefits of short-chain fatty acids and their receptors in inflammation and carcinogenesis. Pharmacol. Ther. 164, 144-151. doi: 10.1016/j.pharmthera.2016.04.007

Tang, W., Wan, S., Yang, Z., Teschendorff, A. E., and Zou, Q. (2018). Tumor origin detection with tissue-specific miRNA and DNA methylation markers. Bioinformatics 34, 398-406. doi: 10.1093/bioinformatics/btx622

Tang, W. H., Kitai, T., and Hazen, S. L. (2017). Gut microbiota in cardiovascular health and disease. Circ. Res. 120, 1183-1196.

Tang, W. H. W., Li, D. Y., and Hazen, S. L. (2019). Dietary metabolism, the gut microbiome, and heart failure. Nat. Rev. Cardiol. 16, 137-154. doi: 10.1038/ s41569-018-0108-7

Thevenet, J., De Marchi, U., Domingo, J. S., Christinat, N., Bultot, L., Lefebvre, G., et al. (2016). Medium-chain fatty acids inhibit mitochondrial metabolism in astrocytes promoting astrocyte-neuron lactate and ketone body shuttle systems. FASEB J. 30, 1913-1926. doi: 10.1096/fj.201500182

Weber, G. J., Foster, J., Pushpakumar, S. B., and Sen, U. (2018). Altered microRNA regulation of short chain fatty acid receptors in the hypertensive kidney is 
normalized with hydrogen sulfide supplementation. Pharmacol. Res. 134, 157165. doi: $10.1016 / j . p h r s .2018 .06 .012$

Xie, A., Song, J., Lu, S., Liu, Y., Tang, L., and Wen, S. (2021). Influence of diet on the effect of the probiotic lactobacillus paracasei in rats suffering from allergic asthma. Front. Microbiol. 12:737622. doi: 10.3389/fmicb.2021.73 7622

Yang, H., Luo, Y., Ren, X., Wu, M., He, X., Peng, B., et al. (2021). Risk prediction of diabetes: big data mining with fusion of multifarious physical examination indicators. Inform. Fusion 75, 140-149. doi: 10.1016/j.inffus.2021.02.015

Yu, Y., Jia, T. Z., Cai, Q., Jiang, N., Ma, M. Y., Min, D. Y., et al. (2015). Comparison of the anti-ulcer activity between the crude and bran-processed Atractylodes lancea in the rat model of gastric ulcer induced by acetic acid. J. Ethnopharmacol. 160, 211-218. doi: 10.1016/j.jep.2014.10.066

Yue, Y., He, Z., Zhou, Y., Ross, R. P., Stanton, C., Zhao, J., et al. (2020). Lactobacillus plantarum relieves diarrhea caused by enterotoxin-producing Escherichia coli through inflammation modulation and gut microbiota regulation. Food Funct. 11, 10362-10374. doi: 10.1039/d0fo02670k

Zhang, X. Y., Shi, S. P., Shen, J., Zhao, M. Y., and He, Q. N. (2019). Functional immunoregulation by heme oxygenase 1 in juvenile autoimmune diseases. Curr. Gene Ther. 19, 110-116. doi: 10.2174/156652321966619071009 2935

Conflict of Interest: The authors declare that the research was conducted in the absence of any commercial or financial relationships that could be construed as a potential conflict of interest.

Publisher's Note: All claims expressed in this article are solely those of the authors and do not necessarily represent those of their affiliated organizations, or those of the publisher, the editors and the reviewers. Any product that may be evaluated in this article, or claim that may be made by its manufacturer, is not guaranteed or endorsed by the publisher.

Copyright (c) $2022 \mathrm{Sun}, \mathrm{Du}, \mathrm{Fu}, \mathrm{Han}, \mathrm{Li}$ and Ju. This is an open-access article distributed under the terms of the Creative Commons Attribution License (CC BY). The use, distribution or reproduction in other forums is permitted, provided the original author(s) and the copyright owner(s) are credited and that the original publication in this journal is cited, in accordance with accepted academic practice. No use, distribution or reproduction is permitted which does not comply with these terms. 